\title{
Clubes de estudantes: espaços pedagógicos de desenvolvimento de competências de vida e formação de empreendedores sociais
}

\section{Student clubs: pedagogical spaces for the development of life skills and training of social entrepreneurs}

\author{
Liliana Faria*, João Proença** \\ *Universidade Europeia, Laureate International Universities \& SOCIUS/CSG, *,**ISEG-Universidade de Lisboa, \\ **FEP-Universidade do Porto \& Advance/CSG,
}

\begin{abstract}
Resumo
Assente num paradigma que tem por meta a aprendizagem, e não apenas o ensino, e partindo do entendimento de que a Universidade pode, e deve, construir ambientes que favoreçam o desenvolvimento global do estudante, este artigo descreve e mostra como a implementação de Clubes de Estudantes (CE) desenvolve as competências pessoais e sociais dos estudantes. $\mathrm{O}$ artigo analisa o processo de implementação dos CE e as principais atividades de cinco CE universitários e, evidencia que estes são espaços pedagógicos de desenvolvimento de competências de vida e de estímulo ao empreendedorismo, para os estudantes.

Palavras-chave: clubes de estudantes, competências de vida, empreendedorismo social
\end{abstract}

\begin{abstract}
Based on a paradigm that aims to learn, not just teaching, and starting from the understanding that the University can and should build environments that favour the overall development of the student, this article describes and shows how the implementation of Student Clubs (SC) develop students' personal and social skills. The article analyses the implementation process of the SC and the main activities of five university students' clubs and that the SC are pedagogical spaces for developing life skills and stimulating entrepreneurship for students.
\end{abstract}

Keywords: student clubs, life skills, social entrepreneurship.

\section{Introdução}

De acordo com relatório para a UNESCO, da Comissão Internacional sobre Educação para o Século XXI (2000), a educação deve organizar-se em torno de quatro aprendizagens fundamentais: aprender a conhecer, aprender a fazer, aprender a conviver e, aprender a ser.

$\mathrm{O}$ primeiro pilar, aprender a conhecer, diz respeito à aquisição de instrumentos de descoberta, construção e reconstrução de conhecimento. Por sua vez, o segundo pilar, aprender a fazer, remete para a coragem de concretizar, de correr riscos, de errar sem medo, mesmo tentando acertar. Salienta a disponibilidade para estar aberto a reelaborar, trocar e valorizar o espírito cooperativo do trabalho em equipa. O pilar aprender a conviver, supõe a capacidade de aprender a lidar com o desafio da convivência, da cooperação, com respeito a todos, em todas as atividades humanas. Por fim, o último dos quatro pilares, o pilar aprender a ser, diz respeito ao desenvolvimento da sensibilidade, sentido ético e estético, responsabilidade pessoal, pensamento autónomo e crítico, imaginação, criatividade, iniciativa e, consequente crescimento integral da pessoa. Essas quatro aprendizagens, interligadas entre si, são os pilares do conhecimento, e como tal, devem ser desenvolvidas pela prática pedagógica em todos os ciclos de enino (Delors, 2012).

A Universidade, como uma das instituições formadoras de cidadãos, deve assumir a responsabilidade para que tais aprendizagens estejam disponíveis aos estudantes (Delors, 2000; UNESCO, 2015). Contudo, nem sempre isso acontece. De acordo com Delors (2000), no ensino superior, a aprendizagem tende a apoiar-se, sobretudo nos primeiros dois pilares, aprender a conhecer e aprender a fazer, ficando os outros dois pilares, aprender a conviver e aprender a ser, negligenciados, ou subentendidos como prolongamentos naturais dos dois primeiros. Pelo que, assim sendo, o enorme desafio de levar à prática formas inovadoras e motivantes de operacionalização de uma educação global, integrada e estruturada de saberes - conhecer, fazer, conviver e ser - ao longo de toda a vida, é colocado à Universidade (García-Aracil \& Van der Velden, 2008). À Universidade é pedido que assuma um papel mais ativo na oferta de atividades que permitam o desenvolvimento holístico do estudante, compreendendo uma visão integradora de competências pessoais, interpessoais, sociais, e éticas que possibilitem a capacitação do estudante para o desenvolvimento de um melhor raciocínio crítico, 
uma adequada perceção de si, da realidade, do mercado e da sua projeção profissional, mas também para o empreendedorismo e para pleno exercício da cidadania (Bennet, Dunne, \& Carré, 1999; Lans, \& Gulikers, 2010; Tuning, 2003). Acontece, porém, que a aquisição de muitas destas competências passa não tanto pela via do ensino, mas sobretudo pela sua prática através da imersão em ambientes de aprendizagem onde sejam sistematicamente exercitadas e treinadas.

Os Clubes de Estudantes (CE) são, por conseguinte, um desses ambientes de aprendizagem centrados no desenvolvimento global do estudante.

Um CE é um grupo criado, constituído e gerido por estudantes, com o suporte e apoio das estruturas da Universidade (Faria \& Proença, 2017). O objetivo expresso de um CE é o de facilitar, aos seus membros, a aquisição de competências, sejam elas a nível pessoal, social e ético, com o objetivo de serem ser transferidas para outros contextos de vida, que não apenas o académico (Pittawaya, Gazzardb, Shorec, \& Williamsond, 2015), denominadas pela World Health Organization (1997) como competências de vida. Trata-se de competências que facilitam o desenvolvimento de outras capacidades psicológicas indispensáveis para lidar com os desafios da vida, sendo fundamentais para a promoção do desenvolvimento holístico saudável (Danish \& Donohue, 1995; Papacharisis, Goudas, Danish, \& Theodorakis, 2005). As competências de vida não surgem apenas do contacto com diferentes situações e desafios, mas da realização de um conjunto de iniciativas, desde palestras, debates, participação em competições, projetos de serviço comunitário, entre outras (Pittawaya et al., 2015; Wright, Li, Ding \& Pickering, 2010), através da demonstração, modelagem e prática (Kolb, 1984; Kolb, \& Kolb, 2006), conducentes à criação de competências e atitudes empreendedoras (Cope \& Watts, 2000; Politis, 2008).

Os CE contribuem para que os estudantes possam passar por situações diversas, normalmente desafiantes, simuladoras da vida real ou mesmo já integradas na vida e na complexidade económica e social do contexto, de modo a que possam aprender fazendo e desenvolver a autoconfiança, a iniciativa, a assunção de riscos, a criatividade, a resolução de problemas, a comunicação e o trabalho em equipa (Danish, \& Donohue, 1995; Gomes \& Marques, 2013; Petitpas, Van Raalte, Cornelius, \& Presbrey, 2004; Faria \& Proença, 2017), considerados ainda por Heinonen e Poikkijoki (2006) como resultados da educação para o empreendedorismo dos jovens.

\section{Método - Processo de implementação dos Clubes}

A metodologia de implementação dos CE organizou-se em três fases: candidaturas, seleção dos líderes e, constituição dos clubes. Assim sendo, foi criada uma call de candidaturas para líderes de clubes, onde se solicitou aos estudantes que apresentassem uma ideia de um CE que quisessem criar e liderar. Foram rececionadas e avaliadas 22 candidaturas que cumpriam as condições exigida na call, de um universo global de cerca de 6000 estudantes universitários. A avaliação e seleção dos candidatos baseou-se em três critérios: avaliação curricular dos candidatos, pitch da ideia de CE e entrevista. Foram selecionados cinco líderes e, consequentemente, constituídos cinco CE. Os líderes selecionados foram convidados a formar a sua equipa, via recrutamento e seleção e, em conjunto, definirem a missão, visão, valores $\mathrm{e}$, plano de atividades do clube.

As tabelas 1 e 2 apresentam os clubes implementados, caracterizando os membros de cada clube e apresentando as suas missões, respetivamente.

Tabela 1.

Clubes de Estudantes e membros

\begin{tabular}{|c|c|}
\hline Clube & Membros \\
\hline $\begin{array}{c}\text { Clube } \\
\text { A }\end{array}$ & $\begin{array}{l}10 \text { Estudantes ( } 4 \text { raparigas, } 6 \text { rapazes) dos } \\
\text { diferentes anos, dos cursos de Ciências da } \\
\text { Comunicação; Gestão de Empresas; Gestão do } \\
\text { Desporto; Design e; Direito. }\end{array}$ \\
\hline $\begin{array}{c}\text { Clube } \\
\text { B }\end{array}$ & $\begin{array}{l}9 \text { Estudantes ( } 2 \text { raparigas, } 7 \text { rapazes) dos } \\
\text { diferentes anos, dos cursos de Management; } \\
\text { Ciências da Comunicação; Psicologia; Gestão } \\
\text { de Marketing e; Gestão e Estratégica } \\
\text { Empresarial. }\end{array}$ \\
\hline $\begin{array}{l}\text { Clube } \\
\text { C }\end{array}$ & $\begin{array}{l}4 \text { Estudantes (4 raparigas) do } 2^{\circ}, 3^{\circ} \text { anos, dos } \\
\text { cursos de Psicologia e Design. }\end{array}$ \\
\hline $\begin{array}{l}\text { Clube } \\
\text { D }\end{array}$ & $\begin{array}{l}16 \text { Estudantes (2 raparigas, } 7 \text { rapazes) dos } \\
\text { diferentes anos, dos cursos de Turismo, Gestão } \\
\text { de Marketing; Secretariado e Comunicação } \\
\text { Empresarial; Gestão Hoteleira; Gestão; Gestão } \\
\text { de Recursos Humanos e; Marketing e } \\
\text { Publicidade. }\end{array}$ \\
\hline $\begin{array}{c}\text { Clube } \\
\text { E }\end{array}$ & $\begin{array}{l}12 \text { Estudantes (6 raparigas e } 6 \text { rapazes) dos } \\
\text { diferentes anos, dos cursos de Ciências da } \\
\text { Comunicação. }\end{array}$ \\
\hline
\end{tabular}


Tabela 2.

Missão de cada Clube

\begin{tabular}{|c|c|}
\hline Clube & Missão \\
\hline Clube A & $\begin{array}{l}\text { Criar um espaço formal e informal de } \\
\text { discussão para melhorar as capacidades de } \\
\text { comunicação e desenvolver competências } \\
\text { de pensamento crítico sobre questões } \\
\text { atuais e controversas, permitindo-lhes } \\
\text { assumir posições num registo } \\
\text { democrático, responsável, cientificamente } \\
\text { sustentado e com base no respeito, } \\
\text { tolerância e confrontação de ideias. }\end{array}$ \\
\hline Clube B & $\begin{array}{l}\text { Promover um espírito empreendedor entre } \\
\text { os alunos através da identificação, } \\
\text { incubação e implementação de ideias } \\
\text { empresariais. }\end{array}$ \\
\hline Clube C & $\begin{array}{l}\text { Assumir-se como uma rede de apoio ao } \\
\text { estudante na promoção da saúde e bem- } \\
\text { estar académico, físico e mental. }\end{array}$ \\
\hline Clube D & $\begin{array}{l}\text { Promover de eventos de cariz corporativo } \\
\text { e lúdico, de forma a aproximar toda a } \\
\text { comunidade académica e a potenciar a } \\
\text { relação com exterior. }\end{array}$ \\
\hline Clube E & $\begin{array}{l}\text { Criar conteúdos jornalísticos para diversas } \\
\text { plataformas mediáticas. }\end{array}$ \\
\hline
\end{tabular}

Todos os clubes seguiram o princípio associado ao projeto desenvolvido pelos autores de serem "Clubes de Estudantes para Estudantes", ou seja, estudantes a desenvolverem atividades com outros estudantes e para beneficio e desenvolvimento dos próprios estudantes, numa vertente de educação cívica e compromisso com a comunidade académica.

\section{Resultados}

A tabela 3 apresenta os principais projetos/atividades desenvolvidas pelos CE no período decorrido entre a sua apresentação pública oficial (fevereiro de 2017) e o final das atividades letivas (maio de 2017).
Tabela 3.

Principais atividades desenvolvidas pelos Clubes de Estudantes

\begin{tabular}{|c|c|}
\hline Clube & Projetos/Atividades \\
\hline $\begin{array}{c}\text { Clube } \\
\text { A }\end{array}$ & $\begin{array}{l}\text {-Sessões semanais de debates internos do } \\
\text { clube, dedicados à discussão e temas da } \\
\text { atualidade; } \\
\text { - Organização e participação de workshops } \\
\text { dedicados ao aperfeiçoamento de } \\
\text { competências comunicacionais e de } \\
\text { competências retóricas e de argumentação, } \\
\text { para os membros do clube; } \\
\text { - Observação, participação e organização de } \\
\text { debates (e.g., observação participantes no } \\
\text { debate quinzenal da Assembleia da Republica; } \\
\text { observação participante no programa de rádio } \\
\text { da Antena 1, "Radicais livres"; organização e } \\
\text { participação ativa no debate de abertura do } \\
\text { Prémio Universitário Comunicar). }\end{array}$ \\
\hline $\begin{array}{c}\text { Clube } \\
\text { B }\end{array}$ & $\begin{array}{l}\text {-Participação em cursos intensivos de } \\
\text { empreendedorismo (e.g., ICELab - Innovation } \\
\text { and Creativity to Enternerprship); } \\
\text {-Candidaturas a concursos de } \\
\text { empreendedorismo (e.g., McGuire, AGEAS - } \\
4^{\text {o }} \text { classificado; Creative Night - } 1^{\text {o }} \\
\text { classificado). }\end{array}$ \\
\hline $\begin{array}{c}\text { Clube } \\
\text { C }\end{array}$ & $\begin{array}{l}\text { - Organização e dinamização de Workshops } \\
\text { para toda a comunidade de estudantes (e.g., } \\
\text { técnicas de estudo e gestão da ansiedade face } \\
\text { aos exames; Dress code for Job Interview). }\end{array}$ \\
\hline $\begin{array}{c}\text { Clube } \\
\text { D }\end{array}$ & $\begin{array}{l}\text { - Organização de Talks para estudantes; } \\
\text { - Organização de Masterclasses; } \\
\text { - Organização de Galas de Final de Ano; } \\
\text {-Colaboração na organização de eventos } \\
\text { institucionais (e.g., evento anual de } \\
\text { Responsabilidade Social da Universidade). }\end{array}$ \\
\hline $\begin{array}{c}\text { Clube } \\
\text { E }\end{array}$ & $\begin{array}{l}\text {-Organização e participação no Workshop de } \\
\text { Escrita Jornalística; } \\
\text {-Organização e implementação de reportagens } \\
\text { áudio visuais (e.g., Student club versão 0.0; “O } \\
\text { Jamor sou eu”; Fake News) }\end{array}$ \\
\hline
\end{tabular}

A tabela 4 apresenta as perceções de 15 estudantes (7 raparigas e 8 rapazes) acerca das competências desenvolvidas no âmbito da sua participação nos CE. Recorrendo a três grupos de discussão, questionou-se os estudantes, que disponibilizaram-se voluntariamente para integrar os grupos, acerca do impacto que sentiram que a participação nos diferentes $\mathrm{CE}$ teve no seu desenvolvimento/crescimento. 
Tabela 4.

Impacto dos clubes de estudantes no desenvolvimento de competências dos estudantes

\begin{tabular}{|c|c|}
\hline $\begin{array}{l}\text { Auto } \\
\text { conhecimento }\end{array}$ & $\begin{array}{l}\text { "Tive vários desafios ao longo da minha } \\
\text { participação, o que tem sido gratificante, } \\
\text { pois aprendi muito sobre mim, sobre como } \\
\text { lidar com pessoas e sobre o Clube, claro. } \\
\text { Sinto-me mais realizada." - Estudante do } \\
\text { Clube B. } \\
\text { "Permitiu-me pensar sobre os meus } \\
\text { interesses profissionais. Isto foi o mais } \\
\text { importante, pois já mudei os meus } \\
\text { interesses bastantes vezes e acho que agora } \\
\text { finalmente me encontro no caminho certo. } \\
\text { Graças ao clube" - Estudante Clube A. }\end{array}$ \\
\hline Confiança & $\begin{array}{l}\text { "Tem-me ajudado muito em termos da } \\
\text { minha autoestima. Tenho arriscado mais, } \\
\text { saído da minha zona de conforto, porque } \\
\text { sei que mesmo que haja erros pelo } \\
\text { caminho, no final vou melhorar muito." - } \\
\text { Estudante do Clube F. }\end{array}$ \\
\hline $\begin{array}{l}\text { Competências de } \\
\text { relacionamento } \\
\text { interpessoal }\end{array}$ & $\begin{array}{l}\text { "(...) criar novos laços de amizade que } \\
\text { facilitam a troca de informação e que } \\
\text { facilitam todo o processo a nível } \\
\text { estudantil" - Estudante do Clube E. }\end{array}$ \\
\hline $\begin{array}{l}\text { Competências } \\
\text { comunicacionais }\end{array}$ & $\begin{array}{l}\text { "Devido à natureza do meu clube, a } \\
\text { capacidade de argumentação desenvolve a } \\
\text { capacidade de persuasão" - Estudante do } \\
\text { Clube C. }\end{array}$ \\
\hline
\end{tabular}

\section{Discussão}

Uma educação mais empreendedora e focada nos quatro pilares de aprendizagem torna-se uma necessidade e um fator de diferenciação para os estudantes que vão ingressar no mercado de trabalho. Consequentemente é crucial que as instituições de ensino superior possam fornecer atividades pedagógicas promotoras dessas competências.

Neste artigo procurou-se evidenciar, do ponto de vista teórico, e mediante a análise da experiência da criação de cinco CE, que estes se constituem como espaços pedagógicos de desenvolvimento de competências de vida e competências empreendedoras, nos seus membros. Deste modo, os CE parecem ser um excelente meio e instrumento pedagógico para desenvolver as competências de vida, implicando e envolvendo os estudantes na sua própria aprendizagem e em processos de trabalho independentes.

Ao levar a cabo as diferentes atividades, no âmbito dos Clubes, os estudantes ganharam conhecimento sobre as competências ("aprender a conhecer"), aplicaram esses conhecimentos e experienciaram a resolução de problemas da vida real ("aprender a fazer"), trabalharam em equipa ("aprender a conviver"), e desenvolveram o espírito empreendedor, crítico, criativo e ético ("aprender a ser"). A conceção e implementação de atividades em todos os clubes, por iniciativa própria, orientadas e apoiadas pelas estruturas universitárias para satisfazer as necessidades dos seus membros e dos seus pares, estimularam o espírito empreendedor dos mesmos. A participação em CE, além de facilitar o desenvolvimento de atitudes tais como autoconfiança, iniciativa, tomada de risco, capacidade para resolver problemas, facilitou o desenvolvimento de competências de comunicação, a apresentação e transmissão de ideias e o trabalho em equipa (Faria \& Proença, 2017), dimensões consideradas pela WHO (1997) como competências de vida e por Heinonen e Poikkijoki (2006) como resultados da educação para o empreendedorismo.

\section{Conclusão}

Este artigo analisou o papel dos CE como espaços pedagógicos de desenvolvimento de competências de vida e de estímulo ao empreendedorismo. Mostramos que estas estruturas, fundamentalmente abordadas como instrumentos de aprendizagem, e não de ensino, criam e desenvolvem um contexto que favorece o desenvolvimento global do estudante, estimulando as suas competências pessoais e sociais. Analisamos, primeiro, o processo de implementação de cinco clubes, e, depois, as suas atividades $\mathrm{e}$ as perceções dos estudantes acerca das competências desenvolvidas no âmbito dos mesmos. Concluímos que os $\mathrm{CE}$ são espaços pedagógicos que fomentam o desenvolvimento das competências dos estudantes relacionadas com a resolução de problemas da vida real, trabalho em equipa, espírito empreendedor, crítico, bem como o desenvolvimento de atitudes de autoconfiança, iniciativa e tomada de risco. Salienta-se a necessidade de se realizarem outros estudos empíricos que identifiquem e meçam as competências de vida e competências empreendedoras desenvolvidas pelos membros dos clubes no seguimento das suas atividades. Da mesma forma, sugerem-se pesquisas que possam identificar as trajetórias dos alunos dentro dos clubes e a sua relação com as trajetórias de carreira desenvolvidas após a formação. Esta análise pode contribuir para aprofundar a compreensão do papel dos CE na formação holística dos estudantes e no desenvolvimento do empreendedorismo.

\section{Referências}

Bennet, N., Dunne, E., \& Carré, C. (1999). Patterns of core and generic skill provision in higher education. Higher Education, 37, 71-93.

Cope, J. \& Watts, G. (2000). Learning by doing. An exploration of experience, critical incidents and reflection in entrepreneurial learning. International Journal of Entrepreneurial Behaviour and Research, 6, 3, $104-24$.

Danish, S. J., \& Donohue, T. (1995). Understanding media's influence on the development of antisocial and prosocial behaviour. In R. Hampton, P. Jenkins, \& T. Gullota (Eds.), Preventing violence in America (pp. 133156). Thousand Oaks, CA: Sage.

Danish, S., Forneris, T., \& Wallace I. (2005). Sport-based life skills programming in the schools. Journal of Applied School Psychology, 21, 2, 41-62. 
Delors, J. (2010). Educação: um tesouro a descobrir. Relatório para a UNESCO da Comissão Internacional sobre Educação para o Século XXI. São Paulo: Cortez.

Evans, M. D., \& Evans, D. M. (2001). Community Service Project Planning for ASCE Student Chapters/Clubs. Journal of Professional Issues in Engineering Education and Practice, 127, 4, 175-183.

Faria, L. \& Proença, J. (2017). Clubes de Estudantes como espaços de exploração e construção da carreira: alguns dados para reflexão. Comunicação apresentada no Seminário Internacional: Desenvolvimento de Carreira e Aconselhamento num Mundo Plural e Desafiador. Universidade do Minho, Braga.

García-Aracil, A., \& Van der Velden, R. (2008). Competencies for young European higher education graduates: labor market mismatches and their payoffs. Higher Education, 55, 219-239.

Gomes, A. R., \& Marques, B. (2013). Life skills in educational contexts: testing the effects of an intervention programme. Educational Studies. University of Minho. School of Psychology.

Heinonen, J. \& Poikkijoki, S. (2006). An entrepreneurialdirected approach to entrepreneurship education: mission impossible?. Journal of Management Development, 25, 1, 80-94.

Kolb, A. Y., \& Kolb, D. A. (2006). Learning styles and learning spaces: a review of the multidisciplinary application of experiential learning theory in higher education. In R. R. Sims, \& S. J. Sims (Eds.), Learning styles and learning: A key to meeting the accountability demands in education (pp. 45 -92). Nova Iorque: Nova Science Publishers.

Kolb, D. A. (1984). Experiential learning: Experience as the source of learning and development. New Jersey: Prentice-Hall.

Lans, T., \& Gulikers, J. (2010). Assessing entrepreneurial competence in entrepreneurship education and training. In A. Fayolle (Ed.), Handbook of research in entrepreneurship education and training (pp. 54-67). Northampton: Edward Elgar.

Papacharisis, V., Goudas, M., Danish, S., \& Theodorakis, Y. (2005). The effectiveness of teaching a life skills program in a sport context. Journal of Applied Sport Psychology, 17, 247-254.

Petitpas, A. J., Van Raalte, J. L., Cornelius, A. E., \& Presbrey, J. (2004). A life skills development program for high school student athletes. Journal of Primary Prevention, 24, 325-334.

Pittawaya, L.A., Gazzardb, J., Shorec, A., \& Williamsond, T. (2015). Student clubs: experiences in entrepreneurial learning. Entrepreneurship \& Regional Development, 27, 3-4, 127-153. doi: 10.1080/08985626.2015.1014865

Politis, D. (2008). The process of entrepreneurial learning. A conceptual framework. In R.T. Harrison \& C.M. Leitch (eds), Entrepreneurial Learning (pp. 44-71). Abingdon: Routledge.
Tuning Project (2003). Tuning Education Structures in Europe. Final Report, Pilot Project Phase 1.

UNESCO (2015). Educação para a cidadania global preparando alunos para os desafios do século XXI. Brasília: UNESCO.

World Health Organization (1997). Programme Mental Health: Division of Mental Health, Life Skills Education in Schools. Genebra, WHO.

Wright, P. M., Li, W., Ding, S., \& Pickering, M. (2010). Integrating a personal-social responsibility program into a lifetime wellness course for urban high school students: Assessing implementation and educational outcomes. Sport, Education, and Society, 15, 277-298. 